\title{
Variações Cromática e de Matizes do Teste de Pfister em Crianças
}

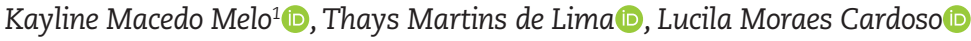 \\ Universidade Estadual do Ceará, Fortaleza-CE, Brasil
}

\section{RESUMO}

Estudos têm sido desenvolvidos com diversos indicadores do Teste das Pirâmides Coloridas de Pfister (TPC), mas as pesquisas sobre as Variações Cromática (VCo) e de Matizes (VMa) ainda são escassas. O objetivo deste trabalho foi analisar as qualidades psicométricas de VCo e VMa do TPC com crianças. Para isso, utilizou-se um banco de dados com 207 protocolos do TPC administrados em estudantes de escolas públicas e particulares, com idades entre 6 e 11 anos. Apresentaram-se as estatísticas descritivas de VCo e VMa conforme as idades das crianças. Além disso, observou-se diminuição da média de VCo conforme o aumento da idade, bem como aumento da amplitude dos valores medianos de VMa nas crianças entre 10 e 11 anos. Sugere-se que essas diferenças podem estar relacionadas a aspectos do desenvolvimento infantil, sendo necessária a continuidade de estudos voltados para o aprimoramento dessas variáveis.

Palavras-chave: teste psicológico; avaliação psicológica; psicometria.

\section{ABSTRACT - Chromatic and Shades Variations of the Pfister Test in children}

Studies have been developed with several indicators of the Pfister Color Pyramid Test (CPT), however, studies related to the Chromatic (CVo) and Shades Variations (SVa) are still scarce. The aim of this study was to analyze the psychometric qualities of the CVo and SVa of the CPT with children. For this, a database was used with $207 \mathrm{CPT}$ protocols administered to students from public and private schools, aged between 6 and 11 years. The descriptive statistics of the CVo and SVa were presented according to the age of the children. A decrease in the mean of the CVo was observed, as well as increased amplitude of the median SVa values in children aged between 10 and 11 years. It is suggested that these differences may be related to aspects of childhood development, and further studies are needed to improve these variables, especially with children.

Keywords: psychological test; psychological assessment; psychometry.

\section{RESUMEN - Variaciones Cromáticas y de Matices del Test de Pfister en niños}

Diversos estudios vienen siendo realizados con respecto a los indicadores del Test de la Pirámide cromática de Pfister (TPC), por otro lado, la investigación sobre las Variaciones Cromáticas (VCo) y Matices (VMa) aún es escasa. El objetivo de este estudio fue analizar las cualidades psicométricas de VCo y VMa de TPC con niños. Para esto, se utilizó una base de datos con 207 protocolos del TPC aplicados a estudiantes de escuelas públicas y privadas, de 6 a 11 años. Se demostraron estadísticas descriptivas de VCo y VMa del de acuerdo con las edades de los niños. Además, hubo una disminución de la media del VCo medida que aumentaba la edad, así como un aumento en la amplitud de los valores medianos de MVa en niños entre 10 y 11 años. Se sugiere que estas diferencias pueden estar relacionadas con aspectos del desarrollo infantil, siendo necesarios más estudios para mejorar estas variables, especialmente para los niños.

Palabras clave: test psicológico; evaluación psicológica; psicometría.

Os testes psicológicos são considerados fontes fundamentais de informação em um processo de Avaliação Psicológica e é responsabilidade das(os) psicólogas(os) a escolha das técnicas que serão utilizadas no processo avaliativo, desde que tais fontes possuam qualidades técnica e científica reconhecidas. No caso de uso dos testes psicológicos, estes devem ter parecer favorável no Sistema de Avaliação de
Testes Psicológicos (Satepsi) do Conselho Federal de Psicologia - CFP (CFP, 2018).

Para ter um parecer favorável para uso profissional, os instrumentos devem atender aos parâmetros psicométricos estabelecidos, isto é, a critérios mínimos de normatização, precisão e validade (CFP, 2018). As normas indicam um referencial a ser seguido pela(o) avaliadora(or) para que os resultados $\mathrm{da}(\mathrm{o})$ examinanda(o) possam ser 
classificados de acordo com os escores do instrumento. Esses escores são os resultados de amostras normativas representativas obtidos por meio das pesquisas científicas, sendo considerados como parâmetro de classificação dos demais sujeitos com características sociodemográficas semelhantes e que venham a ser avaliados pelo mesmo instrumento. É essencial que esse referencial seja rigorosamente definido e descrito durante a execução dessas pesquisas (American Educacional Research Association [AERA]; American Psychological Association [APA]; National Council on Measurement in Education [NCME], 2014).

Para que esses dados normativos sejam confiáveis, é preciso que tenham as evidências de precisão e de validade. A precisão se refere a quanto os resultados do teste são fidedignos, isto é, livre de possíveis erros. A forma de se obter o índice de fidedignidade pode ser concordância entre avaliadores, teste-reteste, consistência interna, formas alternadas ou teste das metades (AERA et al., 2014).

No que concerne à validade, esta diz respeito ao grau em que os escores e as interpretações do teste realmente medem aquilo que foi proposto. Ou seja, a validade refere-se ao quanto a teoria e as evidências sustentam as interpretações do teste para aquilo que ele se propôs a avaliar (AERA et al., 2014). Existem diferentes fontes para buscar evidências de validade, como aquelas baseadas na análise do conteúdo ou domínio, na estrutura interna, nas relações com variáveis externas, em testes avaliando construtos relacionados, em estudos experimentais/quase experimentais e no processo de resposta (CFP, 2018). Para o presente trabalho, será dado enfoque para as evidências de validade baseadas nas relações com outras variáveis, especialmente a do tipo concorrente, a qual diz respeito à obtenção dos escores do teste e das informações de critério de modo simultâneo (AERA et al., 2014).

Dentre os instrumentos psicológicos, há um conjunto de métodos denominados projetivos. Os métodos projetivos possuem estímulos relativamente ambíguos e pouco estruturados (Villemor-Amaral \& Cardoso, 2019; Cardoso \& Resende, 2018; Cardoso \& Villemor-Amaral, 2017; Fensterseifer \& Werlang, 2011), possibilitando a(o) examinanda(o) revelar mais sobre ela(ele) mesma(o) por meio de características subjetivas. Ademais, podem ser utilizados na avaliação da personalidade, da dinâmica emocional e da percepção, por exemplo, desde que se mostrem relevantes para a compreensão do sujeito avaliado (Cardoso \& Villemor-Amaral, 2017).

O Teste das Pirâmides Coloridas de Pfister (TPC) é um exemplo de instrumento projetivo que avalia os aspectos emocionais e, de modo complementar, o desenvolvimento cognitivo da(o) examinanda(o) a partir do modo como são construídos os seus esquemas de pirâmides (Villemor-Amaral, 2015). Desde que foi introduzido no Brasil, várias pesquisas foram realizadas, culminando na elaboração de manuais técnicos a partir delas, incluindo-se os dois mais recentes que se destinam à administração com adultos (Villemor-Amaral, 2012) e com crianças e adolescentes (Villemor-Amaral, 2015).

Tendo em vista as perspectivas de uso do TPC para a avaliação da dinâmica emocional de crianças, realizou-se uma busca nas bases de textos completos da Biblioteca Virtual em Saúde - Psicologia Brasil (BVSPsi Brasil) e dos Periódicos Eletrônicos em Psicologia (Pepsic), as quais levam em consideração as palavras contidas nos resumos, nas palavras-chave e no título dos artigos. Consultaram-se textos completos a partir dos descritores "teste das pirâmides coloridas" e "crianças", conforme sugestão do dicionário de terminologias da BVS-Psi Brasil.

Essa busca inicial tinha o intuito de verificar os estudos existentes sobre esse instrumento e seu uso com o público infantil. Ao todo foram encontrados oito artigos. Quatro que tiveram o objetivo de buscar evidências de validade do TPC para crianças, sendo por meio da comparação entre os sexos (Cardoso, Bessa, \& Targino, 2019), para o uso do TPC com crianças de diversas faixas etárias (Cardoso, Lopes, Marques, \& Targino, 2018), para a utilização do TPC na avaliação da criatividade (Villemor-Amaral, Tavella, Cardoso, Pavan, \& Biasi, 2015b) e para o manejo do TPC com crianças surdas (Cardoso \& Capitão, 2007). Outros dois para verificar os diferentes níveis de maturidade em pessoas de várias idades (Villemor-Amaral, Biasi, Pavan, Tavella, \& Cardoso, 2016) e a influência do sexo, da idade e dos aspectos culturais na seleção das cores no TPC (Villemor-Amaral, Biasi, Cardoso, Pavan, \& Tavella, 2015a). Por fim, outros dois estudos envolveram o uso do TPC concomitante ao HTP (Farah, Cardoso, \& Villemor-Amaral, 2014; Jacob, Loureiro, Marturano, Linhares, \& Machado, 1999).

No levantamento bibliográfico realizado, não foram encontrados estudos recentes sobre os indicadores Variação Cromática (VCo) e Variação de Matizes (VMa). A VCo considera a quantidade de cores escolhidas pela(o) avalianda(o) ao longo das três pirâmides, enquanto a VMa se refere à diversidade de tonalidades utilizadas. Para a análise dessas duas variáveis no TPC, considera-se a existência de 24 tonalidades e oito cores, visto que o branco, preto e cinza são considerados diferentes tonalidades dentro de um mesmo grupo de cores, denominado acromático (Villemor-Amaral, 2012).

Segundo Villemor-Amaral (2012), a VCo e a VMa podem ser consideradas variáveis complementares para a avaliação emocional no TPC. Essas variáveis possibilitam gerar dados, juntamente com os demais indicadores, sobre a expressão das emoções por parte da(o) examinanda(o) (Justo \& Van Kolck, 1976). Entretanto, a relação entre VCo e VMa foi encontrada somente no trabalho de Justo e Van Kolck (1976) com pessoas adultas, no qual os autores identificaram que a média de VCo foi 4,6 em homens e 4,3 em mulheres, ao passo que a média de VMa foi de 7,0 para homens e 6,6 para mulheres. 
Ao comparar essas variações em diferentes faixas etárias, observou-se a média de $\mathrm{VCo}=5,2$ e $\mathrm{VMa}=8,1$ para pessoas de 16 a 22 anos; $\mathrm{VCo}=4,1$ e $\mathrm{VMa}=6,2$ para pessoas de 23 a 29 anos; $\mathrm{VCo}=4,9$ e $\mathrm{VMa}=7,6$ para pessoas de 30 a 44 anos e $\mathrm{VCo}=4,0$ e $\mathrm{VMa}=5,7$ para pessoas de 45 a 65 anos (Justo \& Van Kolck, 1976). Embora não seja a regra, notou-se tendência à diminuição da média de VCo e VMa com o avançar da idade.

Justo e Van Kolck (1976) também descreveram que a média geral da relação entre VCo e VMa é de 4,5 para 6,8 . Segundo esses autores, nos casos em que se observa VCo e VMa inferiores à média esperada, têm-se indicativos de insegurança, limitação, planejamentos prévios, ideias preexistentes e rejeição da multiplicidade. Quando VCo e VMa estão acima da média, sugerem-se elevada riqueza e baixa capacidade inibitória. De modo complementar, Villemor Amaral (1978) indicou que as pessoas que assim procedem seriam mais dispersivas, inconstantes, ansiosas e apresentariam ambição de quantidade, sendo vorazes mesmo que não tenham capacidade de elaboração para assimilar o que procuram absorver.

Quando VCo é baixa e VMa é elevada, pode indicar que a pessoa procede de maneira mais cuidadosa, planejada e segura, bem como limitação sem renúncia à multiplicidade (Justo \& Van Kolck, 1976), possuindo ainda uma tendência a ser mais prudente e cautelosa(o) em relação a suas limitações (Villemor Amaral, 1978). Quando o inverso acontece, isto é, VCo é alta e VMa é baixa, têm-se indicativos de riqueza visando ao que é essencial (Justo \& Van Kolck, 1976), tratando-se de pessoas que têm ambições por quantidade, podendo deixar de levar em consideração a qualidade (Villemor Amaral, 1978). Em suma, observou-se uma relação diretamente proporcional de VCo acima da média esperada à presença de indicadores relativos à maior riqueza, ao passo que uma VMa superior à média sugere maiores características associadas à cautela e segurança. Destaca-se, entretanto, que os autores citaram um estudo de Otfried Spreen (1955, citado por Justo \& Van Kolck, 1976; Villlemor Amaral, 1978), mas não se aprofundaram nas elucidações sobre como identificaram esses dados.

Justo e Van Kolck (1976) também destacaram a análise qualitativa da sequência de VCo e VMa ao longo das três pirâmides. Segundo eles, se VCo e VMa se mantiverem sensivelmente iguais ao longo das pirâmides, há uma sugestão de segurança e tranquilidade no proceder desde o início das atividades. A diminuição progressiva dos valores dessas variáveis da primeira para terceira pirâmide indicaria rejeição, limitação ou perda. O significado atribuído ao aumento progressivo não foi exposto por esses autores.

Justo e Van Kolck (1976) também descreveram os fenômenos chamados "depressão" e "vértice". A depressão é quando o valor da segunda pirâmide é menor que o da primeira e o da terceira, significando perda ou desânimo após alcançar a riqueza e multiplicidade. O vértice ocorre quando a segunda pirâmide tem valores superiores em relação à primeira e à terceira, sugerindo atitude de experimentar. Por fim, os autores mencionaram os "saltos", cujo índice se diferencia demasiadamente dos demais sem apresentar significado para essas situações.

Ainda no que tange ao significado dessas variáveis, baseando-se em sua experiência clínica e nas interpretações teóricas dos indicadores do TPC elaboradas por Villemor-Amaral (2012), Oliveira (2017) expôs que a(o) examinanda(o) possui tendência a dar ênfase na qualidade, em detrimento da quantidade, quando VCo e VMa estão inferiores à média esperada ou quando VCo está abaixo da média e VMa está acima da média. Em contrapartida, quando VCo e VMa estão superiores à média esperada ou quando VCo está alta e VMa está baixa a(o) avalianda $(\mathrm{o})$ tende a dar destaque à quantidade em detrimento da qualidade de sua produtividade.

Oliveira (2017) também mencionou a relação de VCo e VMa com indicadores que avaliam o funcionamento cognitivo, mais precisamente a perseveração cognitiva, relacionada à falta de flexibilidade ou estereotipia no fluxo do pensamento. Esse dado é obtido quando há a mesma quantidade de VCo e VMa nas três pirâmides. Além disso, ressaltou a influência de VCo e VMa na avaliação dos processos defensivos, isto é, dos mecanismos utilizados pela pessoa para aliviar estados de tensão. Para essa análise, é observado se a(o) examinanda(o) utilizou mais cores e tonalidades na primeira pirâmide e foi diminuindo ao longo dos outros esquemas, se aconteceu o inverso, se a quantidade de VCo e VMa foi maior ou menor na segunda pirâmide ou se a pessoa usou a mesma quantidade desses indicadores nos três esquemas (Oliveira, 2017). Destaca-se, entretanto, que não foi realizado estudo de evidências de validade empírica, tratando-se de hipóteses clínicas elaboradas por esse autor a partir dos pressupostos teóricos.

Ao se tratar do uso do TPC para o público infantil, é fundamental que se considerem também aspectos emocionais do desenvolvimento nessa fase da vida. Henri Wallon parte de uma concepção em que a criança evolui de uma forma dinâmica, ou seja, é no contato com outras pessoas, com a cultura e com o contexto em que ela está inserida que vai ocorrer a apropriação de elementos essenciais para o seu desenvolvimento, como a linguagem, a capacidade afetiva, o pensamento, a autoestima, a sensibilidade e o raciocínio. Para Wallon, o desenvolvimento infantil não ocorre de modo contínuo e linear e é marcado por diversas rupturas e retrocessos a cada fase, havendo também uma reorganização de aspectos aprendidos anteriormente e não apenas uma adição de novos elementos (Galvão, 1995).

Segundo Wallon, os fatores biológicos são os que garantem a sequência das fases pelas quais uma criança passa em seu desenvolvimento, embora não determine a homogeneidade no tempo de duração e na idade da criança em cada uma delas, aspectos que podem ter forte influência dos fatores sociais e de interesses individuais 
de cada sujeito. As circunstâncias sociais se tornam cada vez mais ativas nesse processo, sendo responsáveis pelo amadurecimento de habilidades intelectuais mais complexas (Galvão, 1995).

O aprendizado e a expressão das emoções também ocorrem de maneira variada ao longo das diferentes idades da criança, destacando-se que, com o passar dos anos, ela possivelmente irá adquirir cada vez mais a capacidade de expressar-se emocionalmente (Macdonald, KirkPatrick, \& Sullivan, 1996, citado por Cassel, Brunnet, \& Arteche, 2017). Ao nascer, a criança é dotada de uma capacidade inata de expressar-se emocionalmente, seja por meio do choro, da risada, da tristeza etc., e, no decorrer da infância, uma das principais aquisições desse sujeito é a identificação e regulação das emoções, sendo esses aspectos auxiliares para bom desenvolvimento infantil e para um funcionamento mais adaptativo ao meio social (Cassel et al., 2017). Assim, os contextos em que as crianças vivem também são considerados fundamentais para o desenvolvimento e aprimoramento dessa capacidade expressiva (Miguel, Bueno, \& Zuanazzi, 2018).

Usualmente, crianças mais novas possuem maior dificuldade para expressar as suas emoções e, em casos de uma avaliação psicológica com esse público, por exemplo, torna-se essencial o uso de técnicas e testes projetivos que facilitem esse processo de expressão (Cassel et al., 2017). Por ser um instrumento de caráter lúdico, o TPC pode ser utilizado como um recurso para avaliação de aspectos emocionais e cognitivos de crianças em diferentes etapas do desenvolvimento.

Destaca-se, entretanto, que não foram encontrados estudos recentes para os indicadores de VCo e VMa e, na literatura, não foram identificadas pesquisas sobre o uso dessas variáveis com o público infantil. Nesse sentido, esse trabalho trata-se de um estudo que objetivou analisar as qualidades psicométricas das Variações Cromática e de Matizes do TPC no uso com crianças, tendo como hipótese que os valores de VCo e VMa tendem a diminuir com o aumento da idade.

\section{Método}

\section{Participantes}

A amostra da pesquisa foi composta por 207 estudantes de escolas públicas e privadas de Fortaleza-CE. As(os) alunas(os) possuíam idade entre 6 e 11 anos $(M=8,50 ; D P=1,50)$ e escolaridade do $1^{\circ}$ ao $5^{\circ}$ ano do ensino fundamental. A Tabela 1 apresenta os dados referentes à análise estatística descritiva das(os) participantes da pesquisa em função da idade, do sexo e tipo de escola.

Tabela 1

Análise Descritiva das Características Sócio Demográfica das Crianças

\begin{tabular}{|c|c|c|c|c|}
\hline Idade & Sexo & Escola Pública & Particular & Total Geral \\
\hline \multirow{2}{*}{6} & Feminino & 6 & 6 & 12 \\
\hline & Masculino & 5 & 8 & 13 \\
\hline \multirow{2}{*}{7} & Feminino & 13 & 14 & 27 \\
\hline & Masculino & 6 & 3 & 9 \\
\hline \multirow[b]{2}{*}{8} & Feminino & 8 & 12 & 20 \\
\hline & Masculino & 8 & 8 & 16 \\
\hline \multirow{2}{*}{9} & Feminino & 11 & 10 & 21 \\
\hline & Masculino & 10 & 13 & 23 \\
\hline \multirow{2}{*}{10} & Feminino & 14 & 10 & 24 \\
\hline & Masculino & 13 & 15 & 28 \\
\hline \multirow{2}{*}{11} & Feminino & 2 & 5 & 7 \\
\hline & Masculino & 2 & 5 & 7 \\
\hline Total Geral & & 98 & 109 & 207 \\
\hline
\end{tabular}

Os critérios de inclusão na amostra foram a criança estar devidamente matriculada em uma instituição educacional e ter o Termo de Consentimento Livre e Esclarecido (TCLE) assinado por uma(um) das(os) responsáveis. No que se referem aos critérios de exclusão, estes diziam respeito aos sujeitos terem histórico de busca por ajuda psicológica, e/ou psiquiátrica, e de reprovação escolar.

\section{Instrumentos}

Teste das Pirâmides Coloridas de Pfister (TPC). O TPC tem como objetivo avaliar a dinâmica 
emocional e os aspectos cognitivos do sujeito. Durante a administração desse instrumento, solicita-se à pessoa que preencha esquemas de pirâmides com quadrículos coloridos, os quais são distribuídos em oito cores (considerando-se branco, cinza e preto como espectros de uma mesma cor) e 24 tonalidades. Ao final, pede-se que o examinando responda a um inquérito com perguntas sobre a preferência pelas pirâmides construídas e sobre as cores utilizadas. A administração do TPC é individual e considera o modo como a(o) examinanda(o) colocou os quadrículos nas pirâmides, a maneira como ela(ele) executou a atividade e a estrutura ou forma das suas pirâmides (Villemor-Amaral, 2015). Atualmente, o TPC está com parecer favorável para o uso no Satepsi, tanto na versão para adultos quanto para crianças e adolescentes.

\section{Procedimentos}

Para a realização deste estudo, foi utilizado o banco de dados de uma pesquisa realizada entre 2014 e 2016, a qual foi anteriormente aprovada pelo Comitê de Ética em Pesquisa com seres humanos da Universidade Estadual do Ceará (UECE), sob Parecer no 618.913. A coleta de dados aconteceu em escolas públicas e privadas de Fortaleza-CE, sendo realizada após obtenção por escrito da anuência das escolas, do consentimento livre e esclarecido das(os) responsáveis pelas crianças e do assentimento das próprias crianças.

O estudo foi conduzido pelas(os) participantes do Laboratório de Estudos e Práticas em Avaliação Psicológica (Leapsi) da UECE, o qual era composto pela professora responsável pela pesquisa e oito graduandas(os) a partir do $7^{\circ}$ semestre do curso de Psicologia, as(os) quais receberam um treinamento de, aproximadamente, 40 horas para a correta administração e codificação dos instrumentos segundo as orientações contidas nos manuais do teste. Destaca-se que todas(os) as(os) pesquisadoras(es) que auxiliaram na coleta de dados já tinham cursado e sido aprovadas(os) em disciplinas específicas de Avaliação Psicológica.

A coleta de dados foi realizada em uma sessão individual de aproximadamente 60 minutos com cada uma das crianças. Os protocolos com as respostas das crianças foram codificados pelas(os) participantes do Leapsi e, posteriormente, revisados e corrigidos pela professora orientadora da pesquisa.

\section{Análise de Dados}

Todos os protocolos com os dados das crianças foram inseridos em um banco de dados montado no Excel e exportados para o Statistical Package for the Social Sciences (SPSS), versão 23. Foram realizadas, as estatísticas descritivas das Variações Cromáticas e de Matizes das(os) participantes de acordo com a idade, bem como ANOVA para comparar os grupos de variáveis e buscar diferenças significativas em função das faixas etárias.

\section{Resultados e Discussão}

$\mathrm{Na}$ medida em que a idade avança, as crianças tendem a ter melhor capacidade de expressarem suas emoções por meio do TPC (Cardoso et al., 2018; VillemorAmaral et al., 2016; Villemor-Amaral et al., 2015a). Para Villemor-Amaral e Cardoso (2019), ainda que a criança tenha características como vergonha, timidez ou desconhecimento sobre aspectos relacionados a si mesma, os métodos projetivos, como o TPC, não sofrem influência demasiada desses fatores, permitindo obter uma compreensão ampla sobre o seu funcionamento.

Tendo isso em vista, o presente trabalho teve como objetivo analisar as qualidades psicométricas das Variações Cromática (VCo) e de Matizes (VMa) do TPC em crianças, partindo da hipótese de que os valores de VCo e VMa tendem a diminuir com o aumento da idade. Desse modo, ressalta-se a relevância deste estudo na medida em que não foram encontradas pesquisas recentes sobre a VCo e a VMa no TPC (Cardoso et al., 2019; Cardoso et al., 2018; Villemor-Amaral et al., 2016; Villemor-Amaral et al., 2015a; Villemor-Amaral et al., 2015b; Farah et al., 2014; Cardoso \& Capitão, 2007; Jacob et al., 1999) e, nas pesquisas em que essas variáveis foram abordadas (Oliveira, 2017; Justo \& Van Kolck, 1976; Villemor-Amaral, 1978), não foram encontrados dados sobre as qualidades psicométricas para o público infantil.

Considerando que os testes psicológicos devem atender a requisitos mínimos relacionados à normatização, às evidências de validade e à precisão (AERA, APA, \& NCME, 2014; CFP, 2018), inicialmente serão expostas as estatísticas descritivas de VCo e VMa para crianças da capital cearense (Tabela 2) em função da idade em anos. Posteriormente, será apresentada as evidências de validade concorrente por meio da comparação de VCo e VMa por faixas etárias (Tabela 3) e, por fim, serão descritos os tipos de precisão indicados para essas variáveis e os limites para os obter neste estudo.

$\mathrm{Na}$ Tabela 2, foram expostos os dados normativos de VCo e VMa entre as pirâmides de acordo com a idade das crianças. Justo e Van Kolck (1976) propuseram dois tipos de análises para VCo e VMa, sugerindo que seja considerada a sequência das variações em cada uma das três pirâmides e que seja feito o comparativo com os valores esperados, isto é, com os parâmetros normativos. Com relação à análise da sequência das variações em cada uma das três pirâmides, essa deve ser feita de acordo com os dados de cada criança, tratando-se de uma análise qualitativa, o que não convém para o propósito do presente estudo. No que se refere ao comparativo com os valores esperados, é importante que esses parâmetros sejam estabelecidos para que possam ser considerados, entretanto, este foi o primeiro estudo visando estabelecer os valores normativos para VCo e VMa em crianças. Identifica-se, dessa maneira, a necessidade de que estudos semelhantes sejam conduzidos em outros estados 
brasileiros, possibilitando que sejam construídas normas representativas da população brasileira, tal qual consta nos requisitos mínimos esperados para os testes psicológicos no Brasil (CFP, 2018).

Tabela 2

Análise Descritiva de VCo e VMa por Pirâmides de Acordo com a Idade

\begin{tabular}{|c|c|c|c|c|c|c|c|c|c|}
\hline & Idade & VCo P1 & VCo P2 & VCo P3 & VCo M & VMa P1 & VMa P2 & VMa P3 & VMa M \\
\hline \multirow{6}{*}{6} & Média & 6,60 & 6,72 & 7,04 & 6,79 & 12,44 & 11,80 & 12,20 & 12,15 \\
\hline & Desvio Padrão & 1,44 & 1,40 & 1,17 & 1,09 & 2,96 & 3,22 & 1,94 & 2,56 \\
\hline & Percentil 25 & 6,50 & 6,00 & 7,00 & 6,67 & 12,00 & 10,00 & 11,00 & 11,50 \\
\hline & Percentil 75 & 7,00 & 8,00 & 8,00 & 7,50 & 15,00 & 14,50 & 13,50 & 14,33 \\
\hline & Mínimo & 2 & 2 & 3 & 4 & 4 & 2 & 7 & 5 \\
\hline & Máximo & 8 & 8 & 8 & 8 & 15 & 15 & 15 & 15 \\
\hline \multirow{6}{*}{7} & Média & 7,00 & 6,36 & 5,97 & 6,44 & 12,06 & 10,56 & 10,19 & 10,93 \\
\hline & Desvio Padrão & 1,04 & 1,55 & 1,93 & 1,26 & 2,91 & 3,46 & 3,93 & 3,05 \\
\hline & Percentil 25 & 6,25 & 6,00 & 4,25 & 5,33 & 11,00 & 8,25 & 7,25 & 7,75 \\
\hline & Percentil 75 & 8,00 & 8,00 & 8,00 & 7,67 & 14,00 & 13,00 & 13,00 & 13,33 \\
\hline & Mínimo & 5 & 2 & 1 & 4 & 5 & 3 & 1 & 5 \\
\hline & Máximo & 8 & 8 & 8 & 8 & 15 & 15 & 15 & 15 \\
\hline \multirow{6}{*}{8} & Média & 6,81 & 6,75 & 6,39 & 6,65 & 12,11 & 12,19 & 11,86 & 12,06 \\
\hline & Desvio Padrão & 1,70 & 1,46 & 1,81 & 1,40 & 3,66 & 3,50 & 3,94 & 3,16 \\
\hline & Percentil 25 & 6,00 & 6,25 & 6,00 & 6,33 & 10,25 & 10,25 & 9,50 & 10,75 \\
\hline & Percentil 75 & 8,00 & 8,00 & 8,00 & 7,67 & 15,00 & 15,00 & 15,00 & 14,67 \\
\hline & Mínimo & 1 & 3 & 1 & 3 & 3 & 3 & 2 & 4 \\
\hline & Máximo & 8 & 8 & 8 & 8 & 15 & 15 & 15 & 15 \\
\hline \multirow{6}{*}{9} & Média & 6,32 & 6,05 & 6,41 & 6,26 & 11,20 & 10,77 & 11,14 & 11,04 \\
\hline & Desvio Padrão & 2,04 & 1,99 & 1,66 & 1,72 & 4,55 & 4,35 & 3,83 & 3,88 \\
\hline & Percentil 25 & 5,25 & 5,25 & 6,00 & 5,42 & 7,25 & 7,75 & 9,25 & 7,67 \\
\hline & Percentil 75 & 8,00 & 7,00 & 8,00 & 7,59 & 15,00 & 14,00 & 14,00 & 14,00 \\
\hline & Mínimo & 1 & 1 & 1 & 1 & 1 & 1 & 1 & 1 \\
\hline & Máximo & 8 & 8 & 8 & 8 & 15 & 15 & 15 & 15 \\
\hline \multirow{6}{*}{10} & Média & 5,85 & 5,81 & 5,83 & 5,83 & 10,04 & 9,65 & 9,92 & 9,87 \\
\hline & Desvio Padrão & 2,35 & 2,35 & 2,50 & 2,15 & 4,69 & 4,69 & 5,01 & 4,32 \\
\hline & Percentil 25 & 4,25 & 4,00 & 3,25 & 4,33 & 5,25 & 5,00 & 5,00 & 6,33 \\
\hline & Percentil 75 & 8,00 & 8,00 & 8,00 & 7,33 & 14,00 & 14,00 & 14,00 & 13,67 \\
\hline & Mínimo & 1 & 1 & 1 & 1 & 1 & 1 & 1 & 1 \\
\hline & Máximo & 8 & 8 & 8 & 8 & 15 & 15 & 15 & 15 \\
\hline \multirow{6}{*}{11} & Média & 5,64 & 5,64 & 6,21 & 5,83 & 9,79 & 9,64 & 10,21 & 9,88 \\
\hline & Desvio Padrão & 1,95 & 2,34 & 1,25 & 1,65 & 4,89 & 5,06 & 3,17 & 3,88 \\
\hline & Percentil 25 & 4,75 & 4,00 & 5,00 & 4,00 & 4,75 & 4,00 & 8,75 & 5,50 \\
\hline & Percentil 75 & 7,00 & 8,00 & 7,25 & 7,33 & 14,25 & 14,25 & 13,00 & 13,00 \\
\hline & Mínimo & 1 & 1 & 4 & 3 & 2 & 2 & 5 & 4 \\
\hline & Máximo & 8 & 8 & 8 & 8 & 15 & 15 & 15 & 15 \\
\hline \multirow{6}{*}{ Total } & Média & 6,39 & 6,22 & 6,25 & 6,29 & 11,27 & 10,75 & 10,86 & 10,96 \\
\hline & Desvio Padrão & 1,90 & 1,92 & 1,93 & 1,67 & 4,11 & 4,15 & 4,05 & 4,10 \\
\hline & Percentil 25 & 6,00 & 6,00 & 5,00 & 5,33 & 9,00 & 8,00 & 9,00 & 8,33 \\
\hline & Percentil 75 & 8,00 & 8,00 & 8,00 & 7,33 & 15,00 & 14,00 & 14,00 & 14,00 \\
\hline & Mínimo & 1 & 1 & 1 & 1 & 1 & 1 & 1 & 1 \\
\hline & Máximo & 8 & 8 & 8 & 8 & 15 & 15 & 15 & 15 \\
\hline
\end{tabular}

Nota. Variação Cromática na Pirâmide 1 (VCo P1), Variação Cromática na Pirâmide 2 (VCo P2), Variação Cromática na Pirâmide 3 (VCo P3), Média da frequência de Variação Cromática nas três pirâmides (VCo M), Variação de Matizes na Pirâmide 1 (VCo P1), Variação de Matizes na Pirâmide 2 (VCo P2), Variação de Matizes na Pirâmide 3 (VCo P3) e Média da frequência de Variação de Matizes nas três pirâmides (VMa M) 
Após realizar a análise descritiva, foi feita a comparação da média de VCo e de VMa nas três pirâmides em função da idade por meio da ANOVA e não foram encontradas diferenças significativas. Entretanto, ao ponderar o fato de o TPC avaliar a dinâmica emocional e o desenvolvimento emocional da criança não ocorrer de modo linear a cada idade (Galvão, 1995), considerou-se mais adequado efetuar a ANOVA de acordo com faixas etárias. Dessa forma, optou-se por agrupar as crianças a cada dois anos, de modo que o primeiro grupo continha crianças de 6 e 7 anos $(n=61)$, o segundo, de 8 e 9 anos $(n=80)$ e o terceiro, crianças de 10 e 11 anos de idade $(n=66)$. Os dados dessas comparações estão dispostos na Tabela 3.

Tabela 3

Comparação da Média de VCo e VMa nas Três Pirâmides por Faixa Etária

\begin{tabular}{ccccccc}
\hline & Faixa Etária & $M$ & $D P$ & $F$ & P & Eta \\
\hline \multirow{3}{*}{ VCo } & 6 e 7 anos & 6,59 & 1,20 & & 0,02 & 0,03 \\
& 8 e 9 anos & 6,43 & 1,59 & 3,87 & & \\
& 10 e 11 anos & 5,83 & 2,04 & & & 0,01 \\
VMa & 6 e 7 anos & 11,43 & 2,90 & & 0,04 \\
& 8 e 9 anos & 11,50 & 3,59 & 4,38 & & \\
\hline
\end{tabular}

Ao comparar as médias de VCo e VMa entre as faixas etárias (Tabela 3), foram encontradas diferenças significativas tanto para VCo $(p=0,02 ; \mathrm{Eta}=0,03)$ quanto para VMa $(p=0,01 ; \mathrm{Eta}=0,04)$. Observou-se que a média de VCo diminuiu conforme o aumento da idade. A diminuição do VCo com o passar da idade também foi observada no estudo de Justo e Van Kolck (1976), que obteve média de 5,2 para faixa etária entre 16 e 22 anos, de 4,1 para idades entre 23 e 29 anos, de 4,9 para adultos entre 30 e 44 anos e de 4,0 para pessoas com idade entre de 45 e 65 anos. Entretanto, Justo e Van Kolck (1976) pouco exploraram os valores de VCo de modo independente dos valores de VMa, limitando-se a dizer que estaria associada à "riqueza" (Justo \& Van Kolck, 1976, p. 85).

Ao tratar do indicador VMa, os valores praticamente se mantiveram estáveis nas faixas etárias de 6 e 7 anos e de 8 e 9 anos. Houve diminuição da média e aumento do desvio padrão na faixa etária entre 10 e 11 anos, sugerindo uma amplitude maior da média de VMa nessas idades. Justo e Van Kolck (1976) sintetizaram o significado de VMa como "multiplicidade, variedade" (p. 85). Essa amplitude dos valores de VMa em crianças de 10 e 11 anos sugere que qualquer valor entre 4 e 14 estariam de acordo com o esperado.

A variabilidade acentuada nessa faixa etária poderia ser explicada por características próprias presentes no início da adolescência, visto que, segundo Wallon, essa é uma fase que rompe com a estabilidade e tranquilidade afetiva dos estágios anteriores. Wallon acredita que a desorganização desses fatores está ligada a mudanças corporais, complementando que esse é um período em que também há uma predominância de questões afetivas, pessoais, morais e existenciais perpassando a realidade da criança (Galvão, 1995). Ademais, outros aspectos importantes de serem considerados nas avaliações psicológicas e que não foram consideradas neste estudo, envolve o contexto em que a criança está inserida, visto que esse é outro fator que contribui para o desenvolvimento e aprimoramento da capacidade expressiva por parte do público infantil (Miguel et al., 2018; Cassel et al., 2017; Villemor-Amaral et al., 2015a; Cardoso et al., 2019).

Por fim, ao tratar dos estudos de precisão, vale destacar que o TPC é um instrumento de caráter projetivo (Fensterseifer \& Werlang, 2011; Cardoso \& VillemorAmaral, 2017), que avalia a dinâmica emocional de crianças e adolescentes (Cardoso \& Resende, 2018; VillemorAmaral, 2015) e de adultos (Villemor-Amaral, 2012). Considerando as características próprias do instrumento, os estudos de precisão mais indicados são os de concordância entre avaliadores às variáveis que tenham algum grau de subjetividade na interpretação e de teste-reteste (Cardoso \& Villemor-Amaral, 2017).

Considerando que VCo e VMa são atribuídas conforme a frequência de uso de cores e tonalidades e que não há risco de subjetividade na classificação, os estudos de concordância entre avaliadores não seria o mais indicado, ao contrário do estudo de estabilidade temporal, que se apresenta como mais apropriado para esse caso. Destaca-se, entretanto, que o estudo de precisão teste-reteste requer uma abordagem metodológica distinta do que seria possível nesta pesquisa, a qual trabalhou com um banco de dados de material coletado anteriormente.

Esse trabalho analisou aspectos relacionados às qualidades psicométricas de VCo e VMa, sendo relevante também por resgatar as escassas informações publicadas, tal como os estudos de Justo e Van Kolck (1976) e 
Villemor Amaral (1978) sobre esses indicadores. Os dados ora apresentados indicam caminhos promissores à análise dessas variáveis no futuro. Para tanto, novos estudos devem ser desenvolvidos no sentido de aprimorar as informações desses indicadores do TPC, em especial, para o público infantil.

\section{Agradecimentos}

Não há menções.

\section{Financiamento}

Todas as fontes de financiamento para elaboração e produção do estudo (coleta, análise e interpretação dos dados, bem como, escrita dos resultados no presente no manuscrito) foram fornecidas pelo Conselho Nacional de Desenvolvimento Científico e Tecnológico (CNPq), por meio do processo no 408554/2013-4.

\section{Contribuições dos autores}

Todos os autores contribuíram substancialmente para a elaboração do delineamento da pesquisa, análise e interpretação dos dados, bem como, para a revisão textual e aprovação da versão final deste estudo. Todos os autores assumem responsabilidade pública pelo conteúdo do manuscrito.

\section{Disponibilidade dos dados e materiais}

Todos os dados e sintaxes gerados e analisados durante esta pesquisa serão tratados com total sigilo devido às exigências do Comitê de Ética em Pesquisa em Seres Humanos. Porém, o conjunto de dados e sintaxes que apoiam as conclusões deste artigo estão disponíveis mediante razoável solicitação ao autor principal do estudo.

\section{Conflito de interesses}

Os autores declaram que não há conflitos de interesses.

\section{Referências}

American Educacional Research Association [AERA], American Psychological Association [APA], \& National Council on Measurement in Education [NCME] (2014). Standarts for educational and psychological testing. Washington, DC: Autor.

Cardoso, L. M., Bessa, L. B., \& Targino, R. M. (2019). Comparison of the Emotional Indicators of the Pfister Test between Boys and Girls from Ceará-Brazil. Temas em Psicologia, 27(1), 1-10. doi: 10.9788/tp2019.1-01

Cardoso, L. M., \& Capitão, C. G. (2007). Avaliação psicológica de crianças surdas pelo Teste das Pirâmides Coloridas de Pfister. Psico-USF, 12(2), 135-144. doi: 10.1590/S1413-82712007000200002

Cardoso, L. M., Lopes, É. I. X., Marques, T. M., \& Targino, R. M. (2018). Evidências de validade concorrente para uso do Pfister com crianças do Ceará. Psicologia: Teoria e Prática, 20(2), 134-146. doi: 10.5935/19806906/psicologia.v20n2p134-146

Cardoso, L. M., \& Resende, A. C. (2018). O uso dos métodos projetivos na avaliação de crianças. Em M. R. C. Lins, M. Muniz, \& L. M. Cardoso (Orgs.), Avaliação psicológica infantil (pp. 245-264). São Paulo: Hogrefe.

Cardoso, L. M., \& Villemor-Amaral, A. E. (2017). Critérios de cientificidade dos métodos projetivos. Em M. R. Lins \& J. C. Borsa, Avaliação Psicológica: Aspectos teóricos e práticos (pp. 173-186). Rio de Janeiro: Vozes.

Cassel, P. A., Brunnet, A. E., \& Arteche, A. X. (2017). Avaliação infantojuvenil: Emoções, afetos e comportamentos. Em M. R. Lins \& J. C. Borsa, Avaliação Psicológica: Aspectos teóricos e práticos (pp. 236-251). Rio de Janeiro: Vozes.

Conselho Federal de Psicologia [CFP]. (2018). Resolução n. 009/2018. Brasília, DF: Conselho Federal de Psicologia.

Farah, F. H. Z., Cardoso, L. M., \& Villemor-Amaral, A. E. (2014). Precisão e validade do Pfister para avaliação de crianças. Avaliação Psicológica, 13(2), 187-194. Recuperado de: http://pepsic.bvsalud.org/scielo.php?script=sci_arttext\&pid=S1677-04712014000200006\&lng=pt

Fensterseifer, L., \& Werlang, B. S. (2011). Apontamentos sobre o status científico das técnicas projetivas. Em A. E. Villemor-Amaral \& B. S. Werlang, Atualizações em métodos projetivos para avaliação psicológica (pp. 15-36). São Paulo: Casa do Psicólogo.

Galvão, I. (1995). Henri Wallon: Uma concepção dialética do desenvolvimento infantil. Petrópolis: Vozes.

Jacob, A. V., Loureiro, S. R., Marturano, E. M., Linhares, M. B. M., \& Machado, V. L. S. (1999). Aspectos afetivos e o desempenho acadêmico de escolares. Psicologia: Teoria e Pesquisa, 15(2), 153-162. doi: 10.1590/S0102-37721999000200008

Justo, H., \& Van Kolck, T. (1976). O teste das pirâmides de cores. São Paulo: Vetor Editora.

Miguel, F. K., Bueno, J. M. H., \& Zuanazzi, A. C. (2018). Competências socioemocionais de crianças: questões desenvolvimentais. Em M. R. C. Lins, M. Muniz, \& L. M. Cardoso (Orgs.), Avaliação psicológica infantil (pp. 33-49). São Paulo: Hogrefe.

Oliveira, S. E. S. (2017). Pirâmides coloridas de Pfister: Proposta de um sumário interpretativo. Em D. F Pereira \& D. R. Bandeira (Orgs.), Aspectos práticos da avaliação psicológica nas organizações (pp. 45-93). São Paulo: Vetor Editora.

Villemor-Amaral, F. (1978). Pirâmides Coloridas de Pfister. (2 Ed.), Rio de Janeiro: Centro Editor de Psicologia Aplicada LTDA.

Villemor-Amaral, A. E. (2012). As Pirâmides Coloridas de Pfister. São Paulo: Casa do Psicólogo.

Villemor-Amaral, A. E. (2015). As Pirâmides Coloridas de Pfister: Versão para crianças e adolescentes. São Paulo: Casa do Psicólogo.

Villemor-Amaral, A. E., Biasi, F. C., Cardoso, M. L., Pavan, M. P. P., \& Tavella, R. R. (2015a). Rosa e azul: Sexo e idade no Teste de Pfister. Psico-USF, 20(3), 411-420. doi: 10.1590/1413-82712015200304

Villemor-Amaral, A. E., Biasi, F. C., Pavan, P. M. P., Tavella, R. R., \& Cardoso, L. M. (2016). A fórmula cromática no Teste das Pirâmides Coloridas de Pfister em diferentes faixas etárias. Psicologia em Revista, 22(2), 501-515. doi: 10.5752/P.1678-9523.2016V22N2P201

Villemor-Amaral, A. E., \& Cardoso, L. M. (2019). Avaliação da personalidade no Brasil utilizando métodos projetivos. Em M. N. Batista, M. Muniz, C. T. Reppold, C. H. S. S. Nunes, L. F. Carvalho, R. Primi, A. P. P. Noronha, A. G. Seabra, S. M. Wechsler, C. S. Hutz, \& L. Pasquali (Orgs.), Compêndio de Avaliação Psicológica (pp. 475-482). Petrópolis: Vozes. 
Villemor-Amaral, A. E., Tavella, R. R., Cardoso, L. M., Pavan, P. M. P., \& Biasi, F. C. (2015b). Teste das pirâmides coloridas de Pfister e a criatividade em crianças. Revista Psicologia-Teoria e Prática, 16(3), 114-124. Recuperado de http://pepsic.bvsalud.org/scielo.phpscript=sci_ arttext\&pid $=$ S151636872014000300009\&lng $=$ pt\&tlng $=$ pt

\section{Sobre as autoras}

Kayline Macedo Melo é psicóloga formada pela Universidade Estadual do Ceará (UECE) e membro do Laboratório de Estudos e Práticas em Avaliação Psicológica (LEAPSI).

Thays Martins de Lima é estudante de Psicologia na Universidade Estadual do Ceará (UECE) e membro do Laboratório de Estudos e Práticas em Avaliação Psicológica (LEAPSI).

Lucila Moraes Cardoso é psicóloga, mestre e doutora em Psicologia pela Universidade São Francisco (USF). Atualmente, é professora adjunta no curso de Psicologia (UECE) e professora colaboradora no Programa de Pós-Graduação em Psicologia (UFC).

\section{Como citar este artigo}

Melo et al. (2020). Variações Cromática e de Matizes do Teste de Pfister em Crianças. Avaliação Psicológica, 19(3), 342-350. http://dx.doi. org/10.15689/ap.2020.1903.18672.12 